\title{
The Impact of Environmental Light Intensity on Experimental Tumor Growth
}

\author{
MARK A. SUCKOW ${ }^{1}$, WILLIAM R. WOLTER ${ }^{2}$ and GILES E. DUFFIELD ${ }^{3}$ \\ ${ }^{1}$ Department of Veterinary Population Medicine, University of Minnesota, St. Paul, MN, U.S.A.; \\ ${ }^{2}$ Freimann Life Science Center, University of Notre Dame, Notre Dame, IN, U.S.A.; \\ ${ }^{3}$ Department of Biological Sciences, University of Notre Dame, Notre Dame, IN, U.S.A.
}

\begin{abstract}
Background/Aim: Cancer research requires for consistent models that minimize environmental variables. Within the typical laboratory animal housing facility, animals may be exposed to varying intensities of light as a result of cage type, cage position, light source, and other factors; however, studies evaluating the differential effect of light intensity during the light phase on tumor growth are lacking. Materials and Methods: The effect of cage face light intensity, as determined by cage rack position was evaluated with two tumor models using the C57Bl/6NHsd mouse and transplantable B16F10 melanoma cells or Lewis lung carcinoma $(L L C)$ cells. Animals were housed in individuallyventilated cages placed at the top, middle, or bottom of the rack in a diagonal pattern so that the top cage was closest to the ceiling light source, and cage face light intensity was measured. Following a two-week acclimation period at the assigned cage position, animals were subcutaneously administered either $1.3 \times 10^{6}$ B16F10 melanoma cells or $2.5 \times 10^{5}$ Lewis lung carcinoma cells. Weights of excised tumors were measured following euthanasia 18 days (melanoma) or 21 days (LCC) after tumor cell administration. Results: Cage face light intensity was significantly different depending on the location of the cage, with cages closest to the light source have the greatest intensity. Mean tumor weights were significantly less $(p<0.001$ for melanoma; $p \leq 0.01$ for LCC) in middle light intensity mice compared to high and low light intensity mice. Conclusion: The environmental light intensity to which experimental animals are exposed may vary markedly with cage location and can significantly influence experimental
\end{abstract}

Correspondence to: Mark A. Suckow, University of Minnesota, College of Veterinary Medicine, 1365 Gortner Avenue, St Paul, MN 55108, U.S.A. Tel: +1 6126244625, Fax: +1 6126257632, e-mail: msuckowd@umn.edu

Key Words: Experimental variable, cancer model, light intensity, light, tumor, melanoma, mouse. tumor growth, thus supporting the idea that light intensity should be controlled as an experimental variable for animals used in cancer research.

Control of environmental conditions is widely recognized as essential to the use of consistent animal models in cancer research. For example, it is known that the physiology of animals can be profoundly influenced by inconsistent environmental conditions, including temperature, relative humidity, sound, and light exposure and that resulting changes may influence experimental results (1-3). Though light intensity has been demonstrated to be an important variable, studies have examined this effect primarily under conditions that do not examine the range of light intensity that might exist under normal animal housing conditions (4-6). Consequently, the varying intensity of light that might exist under typical housing conditions is seldom considered as an important factor affecting animal health or quality of data. Nonetheless, animals may be exposed to varying intensities of light as a result of cage type, cage position, light source, and other factors (7-9). Because the intensity or brightness of light as a function of the distance from the light source follows an inverse square relationship (10), the distance of an animal from the light may vary considerably between cages.

Regulatory guidance with respect to intensity of light in rooms in which experimental animals are housed is relatively general. For example, ETS No. 123 indicates that bright light should be avoided for some species, and illumination should allow performance of husbandry and inspection of animals (11). The Guide for the Care and Use of Laboratory Animals states that light levels in animals rooms have been established by consideration of light intensities that result in retinopathy of albino rodents (12); and that document further states that for other animals there exist no science-based data that can be used to determine appropriate light intensity levels (12). Such uncertainty with respect to the optimal level of illumination can result in animals being housed under disparate conditions with respect to light intensity, thus representing a potentially important experimental variable. 
The experiments described herein were undertaken to test the hypothesis that the light intensity to which mice used in cancer research are exposed under normal housing conditions represents a variable that may affect experimental results.

\section{Materials and Methods}

Animals. Female, 6-8 week old C57B1/6NHsd specific pathogenfree mice from Envigo, Inc. (Indianapolis, IN, USA) were used in these experiments. Females were used because our laboratory has successfully used this model in female mice in the past. Animals were free of common murine bacterial and viral pathogens, including mouse hepatitis virus, murine parvovirus, polyoma virus, minute virus of mice, ectromelia, lymphocytic choriomeningitis virus, reovirus, Sendai virus, Mycoplasma pulmonis, Pasteurella multocida, Cilia-associated respiratory bacillus, Corynebacterium kutscheri, and Helicobacter hepaticus. In addition, animals were free of Encephalitozoon Cuniculi and ectoparasites. Animals were housed in clear polysulfone cages (JAG ${ }^{\mathrm{TM}}$ model PC75JCLMVSH) that were $1.59 \mathrm{~mm}$ thick, each providing space of $7 " \times 11 " \times 5$ ", on an Allentown 140-cage mouse individually ventilated cage rack (model MDJU140MVSPSHRX; Allentown, Inc., Allentown, NJ, USA) that was calibrated to deliver 50 cage air changes per hour. Mice were allowed ad libitum access to reverse osmosis water delivered via an automatic watering line and provided a standard mouse diet (Teklad Global 16\% Protein Rodent Diet; Teklad, Inc., Madison, WI, USA) via a wire bar cage lid (model WBLEBT; Allentown, Inc., Allentown, NJ, USA). Cages were provided with corn cob bedding (1/4-inch Bed-O-Cob ${ }^{\mathrm{TM}}$, The Andersons, Maumee, $\mathrm{OH}, \mathrm{USA}$ ) but no other enrichment so that dispersal of light would not be interrupted within the cage interior. The light within rooms was provided on a 12:12 light:dark cycle by multiple fluorescent T8-style lights (Lithonia F-32, cool white 4,000 kelvin), one light per ballast, that were recessed into the room ceiling. Though the lights were off at night, the room was not designed to be completely devoid of light contamination. Animal manipulations were all conducted between 08:00 and 11:00 (U.S. Eastern time zone). All animal studies were approved by the University of Notre Dame Institutional Animal Care and Use Committee.

Light exposure and measurement. The light intensity was measured at the interior cage front using a commercial photometer with a 20,000 1x range and an accuracy of $\pm 4 \%$ at $<10,000$ 1x (Handheld LUX Light Meter Photometer Model PLMT21, Pyle, Inc., Brooklyn, NY, USA). Briefly, three readings per cage were taken and the mean calculated and used as the cage-face light intensity in lux. With the light source located in the ceiling along the middle of the room, the cage rack was positioned in the middle of the room, with the following cage rack positions used for this study: top corner of the rack nearest the light source (high light); middle of the rack (medium light); and bottom corner farthest from the light source (low light) (Figure 1). At each position, three adjacent cages were located, with four mice housed in each cage. The mice were allotted two weeks for acclimation to their respective light levels before the tumor induction experiment was initiated.

Tumor induction and measurement. For both $\mathrm{B} 16 \mathrm{~F} 10$ mouse melanoma and LLC models, cells were obtained from American Type Culture Collection (Manassas, VA, USA) and were grown to confluence under $5 \% \mathrm{CO}_{2}$ in Dulbecco's Modified Eagle's Medium (DMEM; Sigma Aldrich, Inc., St. Louis, MO, USA) containing 10\% fetal bovine serum and 1\% Penicillin-Streptomycin (Sigma Aldrich). The cells were removed from the flasks by addition of $0.25 \%$ trypsin-EDTA solution, followed by centrifugation and suspension in DMEM. For the melanoma model, each mouse received $1.3 \times 10^{6}$ viable B16F10 cells administered subcutaneously in the lower flank in a volume of $100 \mu \mathrm{l}$. For the LLC model, each mouse received $2.5 \times 10^{5}$ viable cells subcutaneously in the lover flank in a volume of $100 \mu \mathrm{l}$. Between mice, the cells were re-suspended to ensure that a consistent number of cells were administered. Twelve mice were randomly assigned to each tumor group and light intensity level in all experiments. Following administration of cells, mice were returned to their home cages. Experiments with the LLC model were conducted twice to ensure validity. Mice were euthanized by carbon dioxide narcosis followed by cervical dislocation eighteen days after B16F10 tumor cell administration or 21 days after LLC cell administration. Following euthanasia, the tumor mass was excised, skin and connective tissue trimmed away, and the tumors were weighed on a digital balance. In circumstances where more than one mass was present, all were harvested and weighed as one (13).

Statistical analysis. All tumor weight data is presented as mean \pm standard deviation and were analyzed using one- way ANOVAs followed by Tukey's HSD using the statistical computing program R (R Foundation for Statistical Computing, Vienna, Austria). Differences were considered significant when $p \leq 0.05$.

\section{Results}

Light intensity measurements. Photometric measurement of cage-face light intensity demonstrated significant $(p \leq 0.001)$ differences depending on the location of the cages on the rack. For experiments using the melanoma model, the intensity at the high light location reached $320.8 \pm 7.1 \mathrm{~lx}$ while cages at medium and low light locations were subjected to intensities of $169.0 \pm 16.8 \mathrm{~lx}$ and $3.1 \pm 0.3 \mathrm{~lx}$, respectively. For experiments using the LLC model, the intensity at the high light location was $290+8.21 x$ (first experiment) and 379 $\pm 18.31 x$ (second study), while cages at medium light locations were exposed to intensities of $135 \pm 16.1 \mathrm{~lx}$ (fist study) and 162 $\pm 7.2 \mathrm{~lx}$; and cages located at low light locations were subjected to intensities of $3.0 \pm 0.4 \mathrm{~lx}$ (first study) and 3.0+0.2 1x (second study). Thus, depending on the location of the cage on the rack, mice could be exposed to cage-face light intensities varying by 100 -fold.

Tumor growth. To evaluate the effect of cage-face light intensity on tumor growth, tumors were excised at the time of euthanasia and weighed after connective tissue was trimmed away. As shown in Figure 2, the mean melanoma tumor weight was significantly $(p \leq 0.001)$ greater in mice housed in either low $(5.95 \pm 3.24 \mathrm{~g})$ or high $(6.36 \pm 2.81 \mathrm{~g})$ light intensity cages versus those housed under conditions of medium light intensity $(1.47 \pm 0.88 \mathrm{~g})$. In contrast, there was no significant difference in mean tumor weights for mice housed under conditions of low versus high light intensity. Similarly, Figure 3 shows that mean LLC tumor weights 


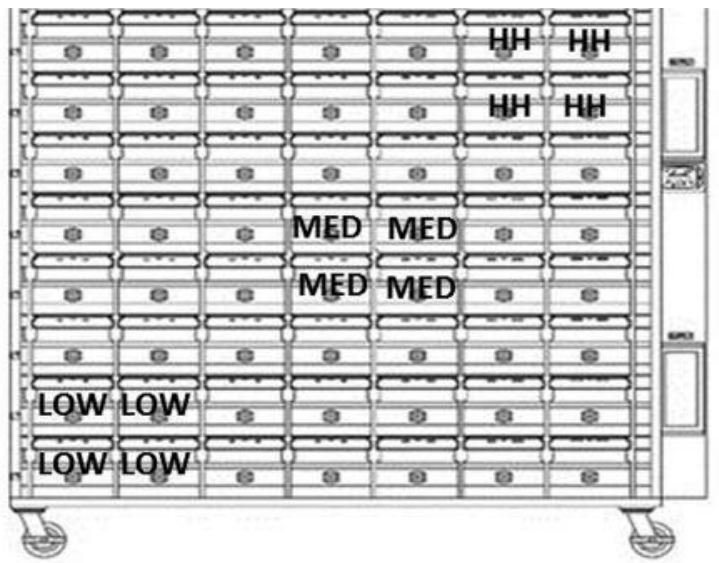

Figure 1. Position of mice on the cage rack with respect to the ceiling fluorescent light shown at the top right of the figure. Cages were placed at locations that were exposed to high (HH), medium (MED), or low (LOW) light intensities. Results from both the mouse melanoma model and the mouse LLC tumor model demonstrated significantly reduced tumor growth in mice housed under conditions of medium light intensity.

were significantly $(p \leq 0.05)$ less for mice housed in medium light intensity $(0.90 \pm 0.68 \mathrm{~g}$ for the first trial and $0.84 \pm 0.66$ $\mathrm{g}$ for the second trial) versus tumors from mice housed under low light intensity $(1.33 \pm 0.79 \mathrm{~g}$ for the first trial and $1.44 \pm 0.83 \mathrm{~g}$ for the second trial) or high light intensity $(1.60 \pm 0.75 \mathrm{~g}$ for first trial and $1.55 \pm 0.24 \mathrm{~g}$ for second trial). The differences between mean tumor weights in mice housed in low versus high light intensity were not significant.

\section{Discussion}

Light exposure has long been recognized as an important variable that can affect the physiological responses of animals. In a research setting, this is particularly important, since data may be skewed by variations in experimental parameters, including light. For example, Dauchy et al. showed that long-term exposure at night to low-intensity red light disrupted the normal circadian melatonin signal as well as other physiologic parameters (14). Pigmented nude rats housed in blue-tinted cages had higher blood melatonin levels during the dark phase compared to rats housed in translucent cages, and this finding was also true for nonpigmented Sprague-Dawley rats housed in amber-, blue-, or red-tinted cages compared to those in translucent cages $(7$, 15). Further, the wavelength of light was found to affect the weights of various endocrine tissues in Hau:ICR mice in a sex-specific manner. For example, the adrenal glands of female mice raised under full spectrum light weighed significantly less than those raised under blue light or black

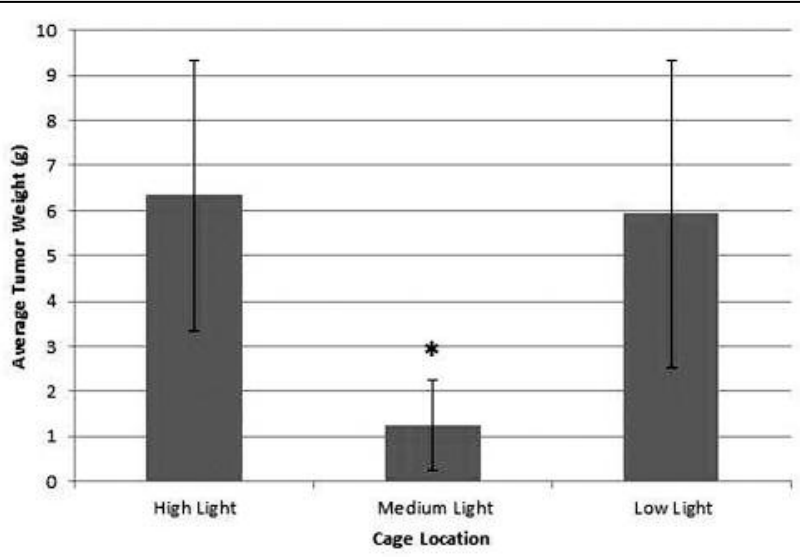

Figure 2. Mean melanoma tumor weights $(g ; \pm$ standard deviation) in C57Bl/6NHsd mice housed under different light intensities based on cage rack position. ANOVA testing revealed significant differences in tumor weight in $C 57$ mice housed on standard racks $(p<0.001)$. The tumors from mice housed under conditions of medium intensity light weighed significantly $(* p \leq 0.001)$ less than those from mice housed under conditions of low or high light intensity. There was no significant difference $(p \geq 0.05)$ in mean tumor weight between mice housed under conditions of low versus high light intensity.

UV light; while the reverse relationship was found for pineal glands; and the thyroid glands of female mice raised under full spectrum light weighed significantly less than those from mice raised under cool white light; in male mice, pituitary glands weighed more when the mice were raised under black UV light versus blue or full spectrum light (16).

Light contamination of animal housing rooms during the dark phase of the light:dark (LD) cycle has been shown to have dramatic effects on animal physiology and experimental outcomes, with significant perturbations in circadian parameters such as circulating melatonin levels; and enhanced growth of tumors in rats, including breast tumor xenografts and hepatomas $(17,18)$. Further, dim light exposure during the dark phase of the LD cycle was demonstrated to drive human breast tumor xenografts in nude rats toward doxorubicin insensitivity (19).

In addition to light wavelength and specific timing of exposure within the LD cycle, intensity of illumination can influence experimental outcomes. For example, rats housed under $10 \mathrm{~lx}$ illumination during the light phase, had reduced heart rates compared to those housed in conditions under $2001 x$ (20). Further, locomotor behavior of rats in an elevated plus-maze was reduced when studies were conducted under light intensities of greater than 1 1x (4).

An important concern regarding light intensity is the effect on animal health, particularly with respect to albino rodents. Exposure of Wistar rats to very high (up to 2,500 lx) during the light phase of the light cycle resulted in necrosis of glandular cells of the Harderian gland, presumably as a result 
of photodynamic action on the porphyrin pigments within the gland (5). The relationship of light intensity to retinal phototoxicity in albino animals has long been recognized, with an early study showing acute retinal damage to animals housed continuously for 24 hours in an environment illuminated by normal fluorescent light (15). Exposure of Sprague-Dawley rats to $270 \mathrm{~lx}$ or greater during the light phase resulted in retinal damage within 7 days, while those maintained at 130 or $65 \mathrm{~lx}$ suffered no such damage (6). Retinal degeneration has been implicated as a source of experimental confounding variable in studies using albino rodents (21); however, our use of pigmented mice was designed to minimize that effect.

Because the intensity of light varies inversely as the square of the distance from the source, it seems apparent that cage location relative to the room light source would result in varying intensities to which cages of animals are exposed. Light intensity was shown to vary greatly (807 1x to $1,3451 \mathrm{x}$ ) within a rodent housing room illuminated by two overhead fluorescent lights; and with cage rack position, with light intensities of 21,43 , and $86 \mathrm{~lx}$ measured for cages on the bottom row, middle row, and top row, respectively (22). Further, Greenman et al. found an increased incidence of retinal atrophy in Balb/c mice housed in cages on the top shelf of a standard rack versus those housed on lower shelves (8).

In the study described here, we tested the hypothesis that mice housed under low intensity as determined by cage rack position would have altered tumor growth compared to animals housed in cages exposed to greater intensity. Our results from two tumor model systems show that mice housed in cages under conditions of medium light intensity grew significantly smaller melanoma and LLC tumors than mice housed in cages exposed to either low or high intensity light. Given the seeming preference of mice for dark conditions, it is unclear why mice housed in the low light intensity group had tumors that were larger than those from mice housed under medium intensity conditions, although it may be that other subtle factors, such as environmental noise or vibration was not distributed evenly across the cage rack. Importantly, it is conceivable that other tumor models may behave differently, however our results emphasize that light intensity as determined by cage rack position is an important variable that should be considered in the care of animals used in cancer research.

To some extent, animals can control their light exposure by locating away from the cage face and by using structural intracage materials such as bedding and nesting material to shield from light; thus, even in circumstances of relatively intense room light, rack positioning away from direct exposure to the light sources and inclusion of materials that allow animals to avoid light exposure would allow for acceptable conditions.

In summary, animal models continue to be essential for research into the pathogenesis and treatment of cancer (23-25). Our results establish that light intensity, as determined by cage rack position, is an important experimental variable in rodent

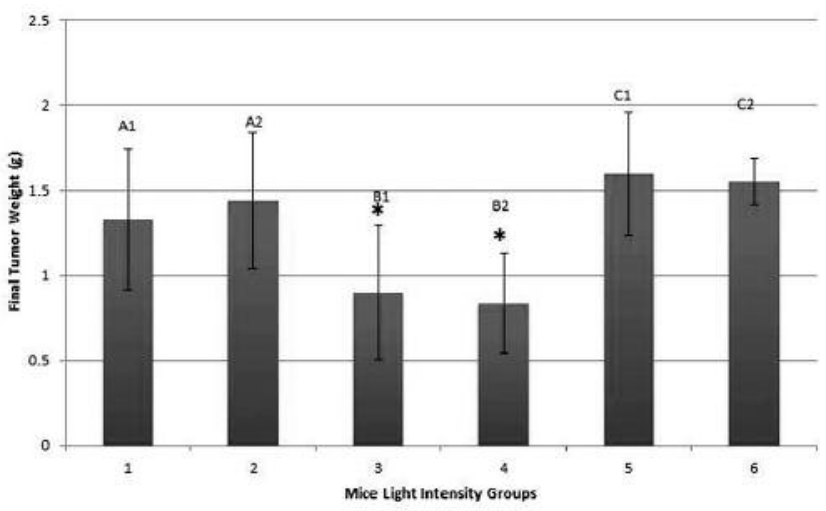

Figure 3. Mean LLC tumor weights ( $g$; \pm standard deviation) in C57Bl/6NHsd mice housed under different light intensities based on cage rack position. Two trials were done for each level of light intensity and are represented as $A 1$ and $A 2$ for the low light intensity trials; B1 and $B 2$ for the medium light intensity trials; and $C 1$ and $C 2$ for the high light intensity trials). The tumors from mice housed under conditions of medium intensity light weighed significantly $\left({ }^{*} p \leq 0.05\right)$ less than those from mice housed under conditions of low or high light intensity. There was no significant difference $(p \geq 0.05)$ in mean tumor weight between mice housed under conditions of low versus high light intensity.

models for cancer research. Because a universally optimal light intensity for research animals remains undetermined, efforts should be directed toward standardizing light intensity within experiments, thus minimizing this as a source of experimental variation.

\section{Acknowledgements}

The Authors wish to acknowledge the contributions of Samuel Wilhite and James Haubert to this work. The work was supported from a grant to GED from the National Institute of General Medical Sciences (R01-GM087508).

\section{References}

1 Clough G: Environmental effects on animals used in biomedical research. Biol Rev 57: 487-523, 1982.

2 Milligan SR, Sales GD and Khirnykh: Sound levels in rooms housing laboratory animals: an uncontrolled daily variable. Physiol Behav 53: 1067-1076, 1993.

3 Nevalainen T: Animal husbandry and experimental design. ILAR J 55: 392-398, 2014.

4 Garcia AMB, Cardenas FP and Morato S: Effect of different illumination levels on rat behavior in the elevated plus-maze. Physiol Behav 85: 265-270, 2005.

5 Kurisu K, Sawamoto O, Watanabe H and Ito A: Sequential changes in the harderian gland of rats exposed to high intensity light. Lab Anim Sci 46: 71-76, 1996.

6 Semple-Rowland SL and Dawson WW: Retinal cyclic light damage threshold for albino rats. Lab Anim Sci 37: 289-298, 1987. 
7 Dauchy RT, Wren MA, Dauchy EM, Hanifin JP, Jablonski MR, Warfield B, Brainard GC, Hill SM, Mao L, Dupepe LM, Ooms TG, and Blask DE: Effect of spectral transmittance through redtinted rodent cages on circadian metabolism and physiology in nude rats. J Am Assoc Lab Anim Sci 52: 745-755, 2013.

8 Greenman DL, Bryant P, Kodell RL, and Sheldon W: Influence of cage shelf level on retinal atrophy in mice. Lab Anim Sci 32: 353-356, 1982.

9 Wren MA, Dauchy RT, Hanifin JP, Jablonski MR, Warfield B, Brainard GC, Blask DE, Hill SM, Ooms TG, and Bohm RP Jr.: Effect of different spectral transmittances through tinted animal cages on circadian metabolism and physiology in SpragueDawley rats. J Am Assoc Lab Anim Sci 53: 44-51, 2014.

10 Ryer AD: The light measurement handbook. Peabody, International Light Technologies Corp., p. 25, 1997.

11 Council of Europe: Appendix A of the European Convention for the Protection of Vertebrate Animals Used for Experimental and Other Scientific Purposes (ETS No. 123). https:// www.aaalac.org/about/ AppA-ETS123.pdf. (2006, Accessed June 19, 2017).

12 Institute for Laboratory Animal Research: Guide for the care and use of laboratory animals, 8th ed. Washington (DC), National Academies Press, pp. 48-49, 2011.

13 Tamayko MM and Reynolds CP: Determination of subcutaneous tumor size in athymic (nude) mice. Cancer Chemother Pharmacol 24: 148-154, 1989.

14 Dauchy RT, Wren MA, Dauchy EM, Hoffman AE, Hanifin JP, Warfield B, Jablonski MR, Brainard GC, Hill SM, Mao L, Dobek GL, Dupepe LM, and Blask DE: The influence of red light exposure at night on circadian metabolism and physiology in Sprague-Dawley rats. J Am Assoc Lab Anim Sci 54: 40-50, 2015

15 Noell WK, Walker VS, Kang BS, and Berman S: Retinal damage by light in rats. Invest Opthalmol 5: 450-473, 1966.

16 Saltarelli CG and Coppola CP: Influence of visible light on organ weights of mice. Lab Anim Sci 29: 319-322, 1979.

17 Dauchy RT, Dauchy EM, Tirrell RP, Hill CR, Davidson LK, Greene MW, Tirrell PC, Wu J, Sauer LA, and Blask DE: Darkphase light contamination disrupts circadian rhythms in plasma measures of endocrine physiology and metabolism in rats. Comp Med 60: 348-356, 2010.
18 Dauchy RT, Dupepe LM, Ooms TG, Dauchy EM, Hill CR, Mao L, Belancio VP, Slakey LM, Hill SM, and Blask DE: Eliminating animal facility light-at-night contamination and its effect on circadian regulation of rodent physiology, tumor growth, and metabolism: a challenge in the relocation of a cancer research laboratory. J Am Assoc Lab Anim Sci 50: 326336, 2011.

19 Xiang S, Dauchy RT, Hauch A, Mao L, Yuan L, Wren MA, Belancio VP, Mondal D, Frasch T, Blask DE, and Hill SM: Doxorubicin resistance in breast cancer is driven by light at night-induced disruption of the circadian melatonin signal. $\mathrm{J}$ Pineal Res 59: 60-69, 2015.

20 Azar TA, Sharp JL, and Lawson DM: Effect of housing rats in dim light or long nights on heart rate. J Am Assoc Lab Anim Sci 47: 25-34, 2008.

21 De Vera Mudry MC, Kronenberg S, Komatsu S-I, and Aguirre GD: Blinded by the light; retinal phototoxicity in the context of safety studies. Toxicol Pathol 41: 813-825, 2013.

22 Bellhorn RW: Lighting in the animal environment. Lab Anim Sci 30: 440-450, 1980

23 Yano S, Takehara K, Kishimoto H, Tazawa H, Urata Y, Kagawa S, Fujiwara T, Bouvet M, and Hoffman RM: Comparison of tumor recurrence after resection of highly- and poorly-metastatic triple-negative breast cancer in orthotopic nude-mouse models. Anticancer Res 37: 57-60, 2017.

24 Rubio CA: Three pathways of colonic carcinogenesis in rats. Anticancer Res 37: 15-20, 2017.

25 Antoniou E, Antonios Margonis G, Angelou A, Zografos GC, and Pikoulis E: Cytokine networks in animal models of colitisassociated cancer. Anticancer Res 35: 19-24, 2015.
Received July 6, 2017

Revised July 25, 2017

Accepted August 2, 2017 